University of Nebraska - Lincoln

DigitalCommons@University of Nebraska - Lincoln

Nutrition and Health Sciences -- Faculty

Publications

Nutrition and Health Sciences, Department of

2017

\title{
Effects of fatigue and recovery on electromechanical delay during isokinetic muscle actions
}

\author{
Cory M. Smith \\ University of Nebraska - Lincoln, cmsmith7@utep.edu \\ Terry J. Housh \\ University of Nebraska-Lincoln, thoush1@unl.edu \\ Ethan C. Hill \\ University of Nebraska-Lincoln, ethan.hill@ucf.edu \\ Josh L. Keller \\ University of Nebraska - Lincoln, jkeller@unl.edu \\ Glen O. Johnson \\ University of Nebraska-Lincoln
}

See next page for additional authors

Follow this and additional works at: https://digitalcommons.unl.edu/nutritionfacpub

Part of the Human and Clinical Nutrition Commons, Molecular, Genetic, and Biochemical Nutrition Commons, and the Other Nutrition Commons

Smith, Cory M.; Housh, Terry J.; Hill, Ethan C.; Keller, Josh L.; Johnson, Glen O.; and Schmidt, Richard J., "Effects of fatigue and recovery on electromechanical delay during isokinetic muscle actions" (2017).

Nutrition and Health Sciences -- Faculty Publications. 195.

https://digitalcommons.unl.edu/nutritionfacpub/195

This Article is brought to you for free and open access by the Nutrition and Health Sciences, Department of at DigitalCommons@University of Nebraska - Lincoln. It has been accepted for inclusion in Nutrition and Health Sciences -- Faculty Publications by an authorized administrator of DigitalCommons@University of Nebraska Lincoln. 


\section{Authors}

Cory M. Smith, Terry J. Housh, Ethan C. Hill, Josh L. Keller, Glen O. Johnson, and Richard J. Schmidt 


\title{
Effects of fatigue and recovery on electromechanical delay during isokinetic muscle actions
}

\author{
Cory M. Smith, Terry J. Housh, Ethan C. Hill, \\ Joshua L. Keller, Glen O. Johnson, \\ and Richard J. Schmidt \\ University of Nebraska-Lincoln \\ Corresponding author - C. M. Smith, email CSmith@unl.edu \\ ORCID iDs \\ Cory M. Smith https://orcid.org/0000-0001-9581-1225 \\ Joshua L. Keller https://orcid.org/0000-0003-0756-9358
}

\begin{abstract}
Objective: To examine muscle-specific differences and the effects of fatigue and recovery on electromechanical delay (EMD) during maximal isokinetic muscle actions.

Approach: Thirteen men performed maximal isokinetic knee extension muscle actions at $60^{\circ} \mathrm{s}^{-1}$, pretest, posttest, and after 5 min of recovery from 25 maximal isokinetic knee extensions. The onsets of the electromyographic, mechanomyographic, and force signals were used to identify EMD measures from the vastus lateralis (VL), vastus medialis (VM), and rectus femoris (RF).

Main results: There were posttest increases in all EMD measures for all muscles that returned to pretest levels after 5 min of recovery. There were, however, no differences in EMD measures between the VL and VM. All EMD values from the RF were greater than the $\mathrm{VL}$ and $\mathrm{VM}$.
\end{abstract}

Published in Physiological Measurement 38 (2017), pp 1837-1847.

doi 10.1088/1361-6579/aa8983

Copyright (c) 2017 Institute of Physics and Engineering in Medicine; published by IOP Publishing. Used by permission.

Submitted 5 May 2017; revised 28 July 2017; accepted for publication 31 August 2017; published 21 September 2017. 
Significance: These findings suggested muscle-specific differences in EMD and that excitation-contraction coupling failure and increased compliance of the series elastic component occurred posttest, but subsided after 5 min of recovery.

Keywords: electromyography, mechanomyography, excitation-contraction coupling, series elastic component

\section{Introduction}

Electromechanical delay (EMD) measures the time delay between the onset of electrical activation of the muscle and the onset of force production (Norman and Komi 1979). Typically (Vos et al 1991, Zhou et al 1998), EMD has been operationally defined as the time period between the onset of the electromyographic (EMG) signal and the onset of force production during a muscle contraction. More recently, however, mechanomyography (MMG) has been used to identify the onset of the lateral oscillations associated with the contraction of skeletal muscle and provides additional information regarding the factors that contribute to EMD (Ce et al 2013, Esposito 2013, Smith et al 2016b). Specifically, the onset of the EMG signal identifies when an electrical impulse activates the muscle while the MMG signal reflects the initiation of movement from the activated muscle fibers (Basmajian and De Luca 1985). The time difference between the onsets of the EMG and MMG signals is a measure of the total duration of the events from the motor unit action potentials travelling along the sarcolemma to cross-bridge formation (excitation-contraction coupling) (Orizio et al 1997). The onset of the MMG signal to the onset of force production is a measure of the time required to take up the muscle-tendon unit slack before force transmission can occur, which has been termed the series elastic component (Orizio et al 1997). Thus, simultaneous assessments of EMG, MMG, and force production allow for the identification of the onset of the EMG signal to the onset of the MMG signal $\left(E M D_{E-M}\right)$, the onset of the MMG signal to the onset of force production $\left(E M D_{M-F}\right)$, and the onset of the EMG signal to the onset of force production (EMD ${ }_{\mathrm{E}-\mathrm{F}}$ ) (Orizio et al 1997, Ce et al 2015a). Therefore, $E M D_{E-M}$ and $E M D_{M-F}$ can measure the relative contributions from excitationcontraction coupling and the series elastic component, respectively, to the overall time duration of $\mathrm{EMD}_{\mathrm{E}-\mathrm{F}}$.

Muscle-specific differences in EMD measures have been controversial in recent research (Hakkinen and Komi 1983, Lieber and Friden 2000, Chan et al 2001, Conchola et al 2013, 2015). For example, Chan et al (2001) reported similar $\mathrm{EMD}_{\mathrm{E}-\mathrm{F}}$ for the vastus lateralis (VL) and vastus medialis (VM) during isometric muscle actions. Conchola et al $(2013,2015)$, however, reported muscle-specific differences in $\mathrm{EMD}_{\mathrm{E}-\mathrm{F}}$ from the $\mathrm{VL}$ and biceps femoris 
during isometric muscle actions. In addition, Hakkinen and Komi (1983) reported muscle-specific differences between the $\mathrm{VL}, \mathrm{VM}$, and rectus femoris (RF) during reflex EMD measures during involuntary muscle actions. Lieber and Friden (2000) suggested that muscles within the human body consist of different muscle-tendon ratios, pennation angles, muscle architecture, contraction velocities, muscle fiber lengths, and muscle fiber-type composition which may affect EMD measurements. Thus, these anatomical and physiological differences may explain the muscle-specific differences in EMD measure. Few studies (Smith et al 2016b, 2017), however, have simultaneously examined $\mathrm{EMD}_{\mathrm{E}-\mathrm{M}^{\prime}}, \mathrm{EMD}_{\mathrm{M}-\mathrm{F}}$ and $\mathrm{EMD}_{\mathrm{E}-\mathrm{F}}$ from muscles within the same muscle group before (pretest), after (posttest), as well as during recovery from the same fatiguing protocol. Therefore, simultaneously examining $\mathrm{EMD}_{\mathrm{E}-\mathrm{M}^{\prime}}$ $\mathrm{EMD}_{\mathrm{M}-\mathrm{F}}$ and $\mathrm{EMD}_{\mathrm{E}-\mathrm{F}}$ from the $\mathrm{VL}, \mathrm{VM}$, and RF may explain the influences of these anatomical and physiological differences on excitation-contraction coupling $\left(E M D_{E-M}\right)$ and the series elastic component $\left(E M D_{M-F}\right)$ prior to and following a fatiguing task.

Fatigue-induced increases in voluntary EMD measures are thought to be influenced by a number of factors including: (1) the buildup of metabolic byproducts (Zhou et al 1998, Begovic et al 2014, de Ste Croix et al 2015, Ce et al 2015a), (2) $\mathrm{Ca}^{2+}$ efflux from the sarcoplasmic reticulum (Zhou et al 1998, Ce et al 2013, Begovic et al 2014), (3) cross-bridge cycling rate (Ce et al 2013, Begovic et al 2014), and (4) increases in muscle temperature (Zhou et al 1998, Ce et al 2013). Thus, metabolic factors related to excitation-contraction coupling as well as exercise-induced increases in the compliance of the series elastic component, lengthen EMD measures (Zhou et al 1998, Ce et al 2013). It has been suggested (Ce et al 2013, Smith et al 2016b, 2017) that fatigue-related excitation-contraction coupling failure results in an increase in $\mathrm{EMD}_{\mathrm{E}-\mathrm{M}^{\prime}}$ while increases in the compliance of the series elastic component associated with muscle temperature result in increased $\mathrm{EMD}_{\mathrm{M}-\mathrm{F}}$. The effects of fatigue on these aspects of voluntary EMD measures, however, have primarily been examined using pre-fatigue versus post-fatigue measurements (Taylor et al 1997, Chan et al 2001, Ce et al 2013). The few studies (Conchola et al 2013, 2015, Rampichini et al 2014) that have examined the recovery of EMD performed isometric (maximal and submaximal) or stimulated muscle actions. Stimulated muscle actions have lower EMD values than those during voluntary muscle actions and stimulated muscle actions do not reflect the motor unit control strategies used to voluntarily contract a muscle (Hopkins et al 2007). In addition, elongation of EMD measures and its process of recovery may be of interest to clinical and athletic setting due to its relation to fatigue, joint instability, injury risk, and recovery times (Minshull et al 2012, Hannah et al 2014, de Ste Croix et al 2015). For example, de Ste Croix et al (2015) reported that increased EMD measures are associated with increased 
risk for ACL injuries in athletes. Minshull et al (2012) and Hannah et al (2014) also indicated that fatigue resulted in greater EMD measures and that during fatigue EMD measures increased, but returned to normal values during recovery. Therefore, it is likely that during the recovery EMD measures will return to normal values. In addition, it has been suggested (Howatson et al 2009, Lacourpaille et al 2013, Smith et al 2017) that EMD measures can be influenced by the intensity and mode (isometric versus dynamic) of a muscle action. Therefore, the purposes of the present study were to examine: (1) the effects of fatigue and recovery on $\mathrm{EMD}_{\mathrm{E}-\mathrm{M}^{\prime}} \mathrm{EMD}_{\mathrm{M}-\mathrm{F}}$ and $E M D_{\mathrm{E}-\mathrm{F}}$ from the $\mathrm{VL}, \mathrm{VM}$, and RF muscles; and (2) the relative contributions from $\mathrm{EMD}_{\mathrm{E}-\mathrm{M}}$ and $\mathrm{EMD}_{\mathrm{M}-\mathrm{F}}$ to $\mathrm{EMD}_{\mathrm{E}-\mathrm{F}}$ from the $\mathrm{VL}, \mathrm{VM}$, and RF. It was hypothesized that there would be fatigue induced-increases in $\mathrm{EMD}_{\mathrm{E}-\mathrm{M}^{\prime}} \mathrm{EMD}_{\mathrm{M}-\mathrm{F}^{\prime}}$ and $\mathrm{EMD}_{\mathrm{E}-\mathrm{F}}$ which would recover after $5 \mathrm{~min}$ of rest. In addition, it was hypothesized that the relative contributions from $E M D_{E-M}$ and $E M D_{M-F}$ to $E M D_{E-F}$ would remain similar during all maximal isokinetic muscle actions.

\section{Methods}

\subsection{Participants}

Thirteen men (mean \pm SD age $24 \pm 3.8$ years; body mass $79.8 \pm 9.7$ kg; height $172.8 \pm 8.6 \mathrm{~cm}$ ) volunteered to participate in this study. The participants were recreationally trained (greater than 6 months of resistance training three times per week), and free from any musculoskeletal injuries or neuromuscular disorders. This study was approved by the Institutional Review Board, and all participants signed a written informed consent and completed a health history questionnaire prior to participation. In addition, this study was performed in agreement with the ethical principles stated in the Declaration of Helsinki (WMA 2013).

\subsection{Experimental approach}

The study consisted of two visits, separated by at least $48 \mathrm{~h}$. The first visit was a familiarization visit which consisted of maximal and submaximal isokinetic knee extension muscle actions. Emphasis was placed on contracting and relaxing as quickly as possible on command. This was performed until participants were comfortable performing these muscle actions. During both visits, participants were able to visualize their muscle activation (EMG and MMG signal) and force on a monitor placed in front of them. The visualization of the muscle actions was used to emphasize the importance of contracting and relaxing as quickly as possible. 
During the testing visit (visit 2) the participants performed two pretest maximal isokinetic knee extension muscle actions at $60^{\circ} \mathrm{s}^{-1}$ with the dominant knee. The participants then performed 25 maximal isokinetic knee extension muscle actions at $60^{\circ} \mathrm{s}^{-1}$. Immediately following the 25 fatiguing isokinetic knee extension muscle actions, each subject performed a posttest maximal isokinetic knee extension muscle action at $60^{\circ} \mathrm{s}^{-1}$. After $5 \mathrm{~min}$ of recovery each subject performed a recovery maximal isokinetic knee extension muscle actions at $60^{\circ} \mathrm{s}^{-1}$.

\subsection{Protocol}

A warmup consisting of five to seven isokinetic knee extension muscle actions were performed at approximately 50 to $70 \%$ of their maximal effort. Following the warmup, each subject performed two maximal isokinetic knee extension muscle actions at $60^{\circ} \mathrm{s}^{-1}$ with $1 \mathrm{~min}$ of rest between the pretest muscle actions. The highest torque value of the two trials was used for the analyses. All isokinetic muscle actions were performed on a Cybex II isokinetic dynamometer calibrated per the Cybex User's Guide (Cybexll 1991). Each participant began each isokinetic knee extension at a joint angle of $90^{\circ}$ and performed the isokinetic knee extension until their leg was fully extended, then immediately back to the starting position of $90^{\circ}$. A miniscule pause was performed between each knee extension where the participants were instructed to relax until force reached zero and there were no EMG or MMG activity on the monitor.

After the pretest muscle actions, participants were given 2 min of rest and then performed the fatiguing protocol consisting of 25 maximal isokinetic knee extension muscle actions at $60^{\circ} \mathrm{s}^{-1}$. Immediately after the fatiguing protocol, the participants performed a maximal isokinetic knee extension muscle action at $60^{\circ} \mathrm{s}^{-1}$ followed by a 5 min recovery period and then another maximal isokinetic knee extension muscle action at $60^{\circ} \mathrm{s}^{-1}$. Electromyography, MMG, and force were simultaneously collected from the $V L, V M$, and RF during each assessment. Each participant was verbally instructed by "Ready, Go!" for when to perform each knee extension throughout the maximal testing and fatiguing protocol.

\subsection{Electromyographic, mechanomyographic, and force signal acquisition}

Bipolar surface electrode arrangements (Ag/AgCl, AccuSensor, Lynn Medical, Wixom, MI, USA) were placed on the VL, VM, and RF of the dominant knee (based on kicking preference) with an interelectrode distance of 30

$\mathrm{mm}$. The skin was dry shaven, abraded, and cleaned with isopropyl alcohol 
prior to electrode placement. For the $\mathrm{VL}$, the bipolar electrode arrangements were placed $66 \%$ of the distance between the anterior superior iliac spine (ASIS) and the lateral border of the patella and orientated at a $20^{\circ}$ angle to approximate the pennation angle of the muscle fibers (Hermens et al 1999, Abe et al 2000). For the VM, the bipolar electrode arrangements were placed $80 \%$ of the distance between the ASIS and the joint space in front of the anterior border of the medial collateral ligament and orientated at a $53^{\circ}$ angle to approximate the pennation angle of the muscle fibers (Hermens et al 1999, Smith et al 2016a). For the RF, the bipolar electrode arrangements were placed $50 \%$ the distance between the ASIS and the superior border of the patella (Hermens et al 1999). A reference electrode was placed over the ASIS. The EMG signals were zero-meaned and bandpass filtered (fourth-order Butterworth) at 10-500 Hz. The MMG signal was measured using a triaxial accelerometer (EGAS-FT-10/V05, Measurement Specialties Inc., Hampton, VA) placed between the bipolar electrode arrangement on the $\mathrm{VL}, \mathrm{VM}$, and RF using double-sided adhesive foam tape. The MMG signals were zero-meaned and bandpass filtered (fourth-order Butterworth) at 5-100 $\mathrm{Hz}$. Force was measured using a low-profile pancake load cell (Honeywell Model 41, Morris Plains, NJ) attached to the lever arm behind the shin-pad participants was attached and was filtered at $5 \mathrm{~Hz}$. All signals were simultaneously collected through a BioPac MP150 (BioPac System Inc., Goleta, CA) at a sampling frequency of $10000 \mathrm{~Hz}$. All signal processing and EMD measurements were performed using custom programs written with LabVIEW software (Version 15.0, National Instruments, Austin TX).

\subsection{Electromechanical delay}

The EMD measurements were determined as the time periods from the onset of the EMG signal to the onset of force $\left(E M D_{E-F}\right)$, onset of the MMG signal to the onset of force $\left(E M D_{M-F}\right)$, and the onset of the EMG signal to the onset of the MMG signal $\left(E D_{E-M}\right)$. The onset of EMG, MMG, and force were determined by the condition of three standard deviations (SDs) from the mean baseline noise observed for each signal, determined from $10000 \mathrm{~Hz}$ (Costa et al 2012, Begovic et al 2014, Stock et al 2015) and were selected offline by the primary investigator (CMS) using a custom written LabVIEW program that provided interactive graphical viewing of each signal (Figure 1).

\subsection{Statistical analysis}

A 3 (Muscle: VL, VM, and RF) $\times 3\left(\mathrm{EMD}^{\mathrm{E}} \mathrm{EMD}_{\mathrm{E}-\mathrm{M}^{\prime}} \mathrm{EMD}_{\mathrm{M}-\mathrm{P}}\right.$ and $\left.\mathrm{EMD}_{\mathrm{E}-\mathrm{F}}\right) \times 3$ (Time: pretest, posttest, and 5 min recovery) repeated measures ANOVA was performed. Follow-up two- and one-way repeated measures ANOVAs and 


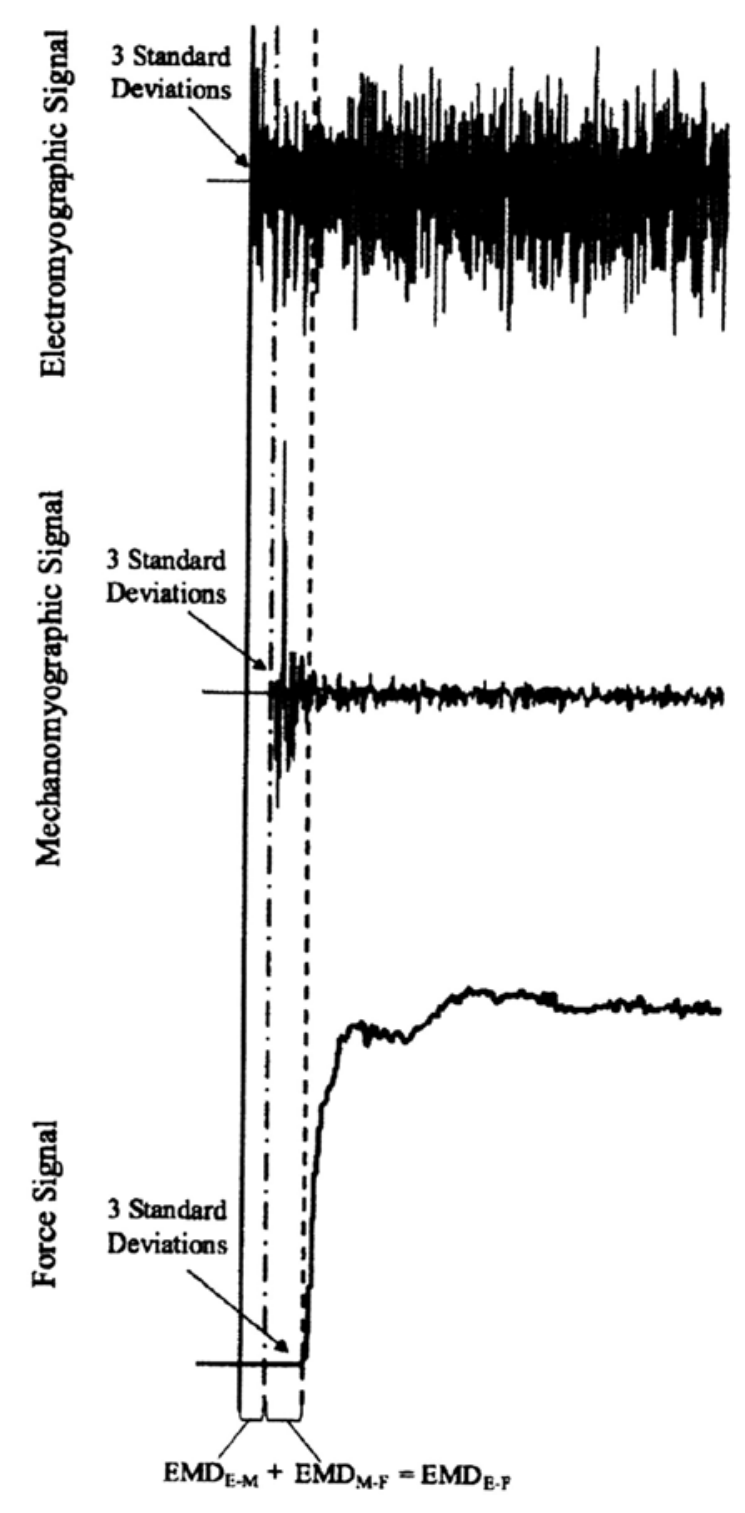

Figure 1. Graphical representation of the electromyographic, mechanomyographic, and force combination for the determinations of electromechanical delay (EMD). Together, these signals allowed for the identification of the onset of the electromyographic signal to the onset of the mechanomyographic signal $\left(E M D_{E-M}\right)$, onset of the mechanomyographic signal to the onset of force production $\left(E M D_{M-F}\right)$, and onset of the electromyographic signal to the onset of force production $\left(E M D_{E-F}\right)$.

paired samples $t$-tests with Bonferonni correction were performed when appropriate. If the assumption of sphericity was violated, the Huynh-Feldt correction was used. An alpha of $p \leq 0.05$ was considered statistically significant for all ANOVAs and Bonferonni significance was based off the number of comparisons made (alpha/n) (SPSS Version 22.0, Armonk, NY). 
Table 1. Electromechanical delay (EMD) measurements (mean and standard error of the mean (SE)) from the vastus lateralis, vastus medialis, and rectus femoris muscles determined from the onset of the electromyographic to mechanomyographic signal $\left(E M D_{E-M}\right)$, onset of the mechanomyographic to force $\left(E \mathrm{ED}_{\mathrm{M}_{-}-\mathrm{s}}\right)$ and onset of the electromyographic signal to the onset of force $\left(E M D_{E-F}\right)$ at pretest, posttest, and 5 min of recovery from the fatiguing workbout during maximal isokinetic knee extension muscle actions at $60^{\circ} \mathrm{s}^{-1}$. All measurements are reported in $\mathrm{ms}$.

\begin{tabular}{|c|c|c|c|}
\hline & $E M D_{E-M}$ & $\mathrm{EMD}_{\mathrm{M}-\mathrm{F}}$ & $E M D_{E-F}$ \\
\hline \multicolumn{4}{|l|}{ Pretest } \\
\hline Vastus lateralisa & $18.71(2.0)$ & $29.95(1.8)$ & $48.66(3.3)$ \\
\hline Vastus medialisa & $23.69(2.5)$ & $31.32(1.9)$ & $55.00(4.1)$ \\
\hline Rectus femorisa,b & $33.13(2.4)$ & $35.86(2.8)$ & $68.99(4.6)$ \\
\hline \multicolumn{4}{|l|}{ Posttest $^{c}$} \\
\hline Vastus lateralis ${ }^{a}$ & 30.65 (1.7) & $41.23(3.7)$ & $71.88(4.8)$ \\
\hline Vastus medialis ${ }^{a}$ & 30.89 (3.3) & $43.52(3.9)$ & $74.41(7.0)$ \\
\hline Rectus femoris ${ }^{a, b}$ & $42.63(3.5)$ & $44.43(3.5)$ & $87.06(7.1)$ \\
\hline \multicolumn{4}{|l|}{$5 \mathrm{~min}$ recovery } \\
\hline Vastus lateralis ${ }^{\mathrm{a}}$ & $24.29(3.5)$ & $32.84(4.1)$ & $57.13(6.4)$ \\
\hline Vastus medialisa & 25.49 (3.7) & 36.98 (4.3) & $62.47(7.1)$ \\
\hline Rectus femorisa,b & 33.18 (4.3) & $36.98(4.4)$ & $70.16(8.7)$ \\
\hline
\end{tabular}

a. $\operatorname{EMDE}-\mathrm{M}<\operatorname{EMD}_{\mathrm{M}-\mathrm{F}}(p<0.01)$.

b. $E M D_{E-M^{\prime}} E M D_{M-p}$ and $E M D_{E-F}$ are greater than those from the $V L$ and $V M$ collapsed across time $(p<0.01)$.

c. Posttest $E M D_{E-\mathrm{M}^{\prime}} E M D_{\mathrm{M}-\mathrm{F}^{\prime}}$ and $E M D_{\mathrm{E}-\mathrm{F}}$ are greater than pretest and 5 min recovery values for each muscle $(p<0.01)$.

\section{Results}

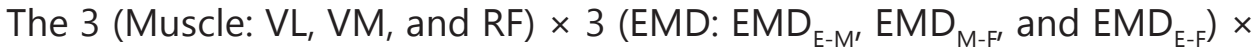
3 (Time: pretest, posttest, and 5 min recovery) repeated measures ANOVA with follow-up two- and one-way ANOVAs as well as post-hoc paired samples $t$-tests indicated no differences in the responses between the VL and $\mathrm{VM}$ for $\mathrm{EMD}_{\mathrm{E}-\mathrm{M}^{\prime}} \mathrm{EMD}_{\mathrm{M}-\mathrm{F}}$ or $E M D_{\mathrm{E}-\mathrm{F}}$ at each time point (pretest, posttest, and 5 min recovery). Therefore, the $\mathrm{VL}$ and $\mathrm{VM}$ responded similarly to one another during the pretest, posttest, and 5 min recovery measurements. That is, there were increases in $\mathrm{EMD}_{\mathrm{E}-\mathrm{M}^{\prime}} \mathrm{EMD}_{\mathrm{M}-\mathrm{P}}$ and $\mathrm{EMD}_{\mathrm{E}-\mathrm{F}}$ from pretest to posttest measurements that returned to pretest values after 5 min of recovery (Table 1; Figure 2).

All EMD values $\left(E M D_{E-M^{\prime}} E M D_{M-F^{\prime}}\right.$ or $\left.E M D_{E-F}\right)$ from the RF were greater than those of the VL and VM at each time-point (pretest, posttest, and 5 min recovery) (Table 1; Figure 2). The RF, however, did have the same pattern of responses for each EMD measure pretest, posttest, and at 5 min recovery (Table 1; Figure 2). Specifically, there were pretest to posttest increases 


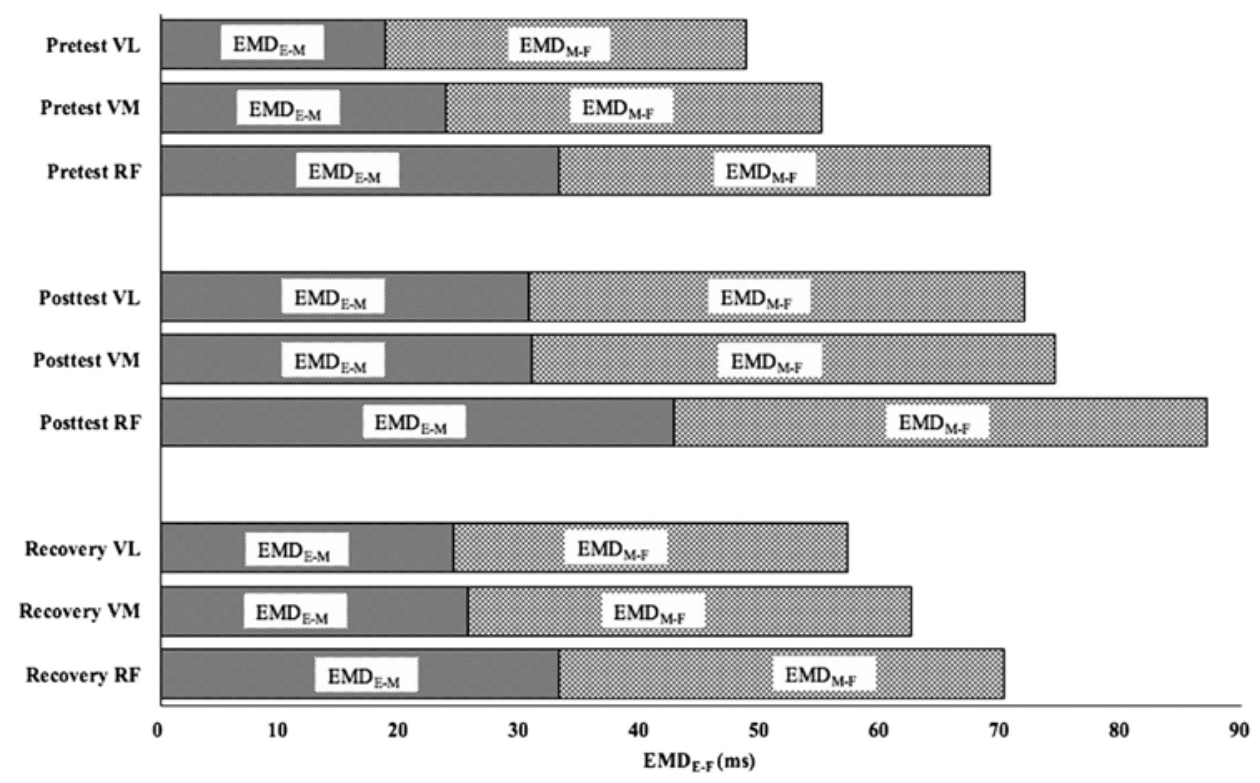

Figure 2. Electromechanical delay (EMD) from the vastus lateralis (VL), vastus medialis (VM), and rectus femoris (RF) from pretest, posttest, and 5 min of recovery measurements during maximal isokinetic knee extension muscle actions at $60^{\circ} \mathrm{s}^{-1}$. The EMD measurements were determined from the onset of the electromyographic signal to the onset of the mechanomyographic signal $\left(E M D_{E-M}\right)$, onset of the mechanomyographic signal to the onset of force production $\left(E M D_{M_{-}}\right)$, and the onset of the electromyographic signal to the onset of force production $\left(\mathrm{EMD}_{\mathrm{E}-\mathrm{F}}\right)$.

in $E M D_{E-M^{\prime}} E M D_{M-F^{\prime}}$ and $E M D_{E-F}$. After 5 min of recovery $E M D_{E-M^{\prime}} E M D_{M-F^{\prime}}$ and $\mathrm{EMD}_{\mathrm{E}-\mathrm{F}}$ returned to pretest values. In addition, at each time-point (pretest, posttest, and 5 min recovery) for all muscles (VL, VM, and RF) $E M D_{E-M}$ was less than $\mathrm{EMD}_{\mathrm{M}-\mathrm{F}}$ and $\mathrm{EMD}_{\mathrm{E}-\mathrm{F}}$ was greater than $\mathrm{EMD}_{\mathrm{E}-\mathrm{M}}$ and $\mathrm{EMD}_{\mathrm{M}-\mathrm{F}}(\mathrm{Ta}-$ ble 1; Figure 2). The following are the descriptive statistics for the strength measures indicated as peak force (mean \pm SD) during the Pretest $(78.6$ $\pm 12.3 \mathrm{~kg})$, Posttest $(52.9 \pm 16.1 \mathrm{~kg})$, and 5 min Recovery $(68.2 \pm 14.7 \mathrm{~kg})$ measurements.

\section{Discussion}

The primary finding of the current study was that $E M D_{E-M^{\prime}} E M D_{M-P}$ and $E M D_{E-}$ ${ }_{F}$ from the $V L, V M$, and $R F$ increased from pretest to posttest, but returned to pretest values after 5 min of recovery during maximal isokinetic knee extension muscle actions (Table 1). These findings were in agreement with those of Conchola et al (2013) who reported a pretest to posttest increase in isometric $\mathrm{EMD}_{\mathrm{E}-\mathrm{F}}(97-122 \mathrm{~ms})$ which returned to pretest values $(99 \mathrm{~ms})$ after 7 
min of recovery from the VL after intermittent $50 \%$ MVIC muscle actions to volitional exhaustion. In addition, Conchola et al (2015) reported an increase in $\mathrm{EMD}_{\mathrm{E}-\mathrm{F}}(91-120 \mathrm{~ms})$ from the VL immediately after a fatiguing protocol at $60 \% \mathrm{MVIC}$ which recovered to pretest values after $7 \mathrm{~min}$ of recovery $(97 \mathrm{~ms}$ ). It has been suggested (Ce et al 2013, 2014, 2015b, Smith et al 2016b, 2017) that increases in $\mathrm{EMD}_{\mathrm{E}-\mathrm{F}}$ reflect peripheral fatigue and can be explained by changes in $E M D_{E-M}$ and $E M D_{M-F}$. Specifically, $E M D_{E-M}$ represents excitationcontraction coupling; a fatigue-induced buildup of metabolic byproducts slows motor unit action potential conduction velocity and causes excitationcontraction coupling failure, which increases $E M D_{E-M}$. The $E M D_{M-F}$ reflects the compliance of the series elastic component which increases with muscle fatigue and, thereby increases $\mathrm{EMD}_{\mathrm{M}-\mathrm{F}}$. Thus, the current study suggested that excitation-contraction coupling failure $\left(E M D_{E-M}\right)$ and increases in the compliance of the series elastic component $\left(E_{M D_{M-F}}\right)$ were evident immediately following the fatiguing, maximal isokinetic protocol. After 5 min of recovery, all EMD measures for all muscles returned to pretest values (Table 1). Therefore, there were fatigue-induced increases in $\mathrm{EMD}_{\mathrm{E}-\mathrm{M}^{\prime}} \mathrm{EMD}_{\mathrm{M}-\mathrm{F}^{\prime}}$ and $E M D_{\mathrm{E}-\mathrm{F}}$ immediately after the fatiguing protocol for all muscles and $5 \mathrm{~min}$ of recovery was sufficient to recover to pretest values. Thus, excitation-contraction coupling failure and increases in the compliance of the series elastic component did not influence any of the EMD measures after 5 min of recovery.

In the current study, there were no significant differences between the $\mathrm{VL}$ and $\mathrm{VM}$ for the $\mathrm{EMD}_{\mathrm{E}-\mathrm{M}^{\prime}}, \mathrm{EMD}_{\mathrm{M}-\mathrm{F}^{\prime}}$ or $\mathrm{EMD}_{\mathrm{E}-\mathrm{F}}$ during the pretest, posttest, or 5 min recovery measurements (Figure 2). The $\mathrm{EMD}_{\mathrm{E}-\mathrm{M}^{\prime}} \mathrm{EMD}_{\mathrm{M}-\mathrm{F}}$ and $\mathrm{EMD}_{\mathrm{E}-\mathrm{F}}$ from the RF, however, were greater than those recorded from the VL and VM during the pretest, posttest, and 5 min of recovery (Figure 2). The findings of the current study were in agreement with those of Chan et al (2001) who reported no differences in $\mathrm{EMD}_{\mathrm{E}-\mathrm{F}}$ measures between the $\mathrm{VL}$ (32.1-52.2 ms) and VM (31.7-48.1 ms) during MVIC muscle actions. These findings, however, were not in complete agreement with those of Vos et al (1991) who reported no differences in $\mathrm{EMD}_{\mathrm{E}-\mathrm{F}}$ measurements (ranging from 95 to 110 $\mathrm{ms}$ ) from the VL, VM, and RF during 50 and $70 \% \mathrm{MVIC}$ muscle actions. The differences in EMD measurements in the current study and those of Vos et al (1991) may indicate intensity- (maximal versus submaximal) and modespecific (isokinetic versus isometric) differences in EMD measures related to the structural differences of the muscles. Specifically, the differences in muscle architecture including muscle length, pennation angle, muscle-to-tendon ratio, and articulation ( $\mathrm{VL}$ and $\mathrm{VM}=$ monoarticulate; $\mathrm{RF}=$ biarticulate) may have contributed to differences in EMD values during different intensities and modes of exercise (Lieber and Friden 2000). In addition, the differences in EMD values may be related to the methodology used to identify the onset of the EMG and force signals (i.e. 3 SD above baseline or a specific 
threshold) as well as EMG, MMG, and force signal conditioning. Thus, during pretest, posttest, and 5 min recovery maximal isokinetic knee extension muscle actions at $60^{\circ} \mathrm{s}^{-1}$ there were muscle-specific (VL and $\mathrm{VM}$ versus RF) differences in the $\mathrm{EMD}_{\mathrm{E}-\mathrm{M}^{\prime}} \mathrm{EMD}_{\mathrm{M}-\mathrm{F}^{\prime}}$ and $\mathrm{EMD}_{\mathrm{E}-\mathrm{F}}$ which may be explained by differences in muscle architecture (Lieber and Friden 2000).

The relative contributions from $E M D_{E-M}$ and $E M D_{M-F}$ to $E M D_{E-F}$ from the $\mathrm{VL}, \mathrm{VM}$, and RF was similar during the pretest, posttest and 5 min recovery measurements, although there were changes in the absolute EMD measures (Table 1). Specifically, excitation-contraction coupling $\left(\mathrm{EMD}_{\mathrm{E}-\mathrm{M}}\right)$ accounted for slightly less than $50 \%$ of the total time delay between the onset of the EMG signal to the onset of force production for the VL (38-43\%), VM (4143\%), and RF (47-49\%) (Table 1). In addition, the time duration to take up the slack of the series elastic component $\left(E \mathrm{DD}_{\mathrm{M}-\mathrm{F}}\right)$ accounted for greater than $50 \%$ of $E M D_{E-F}$ for the VL (57-62\%), VM (57-59\%), and RF (51-53\%) (Table 1). These finding were similar to those of Smith et al (2016b) who reported approximately equal contributions from $\mathrm{EMD}_{\mathrm{E}-\mathrm{M}}$ and $E M D_{\mathrm{M}-\mathrm{F}}$ to $E M D_{\mathrm{E}-\mathrm{F}}$ during pretest and posttest MVIC muscle actions with an increase in absolute EMD after a fatiguing dynamic constant external resistance muscle actions to failure at $70 \%$ of 1 -repetition maximum. Thus, the current and previous study of Smith et al (2016b) suggested that the fatigue-induced buildup of metabolic byproducts $\left(\mathrm{EMD}_{\mathrm{E}-\mathrm{M}}\right)$ and increased compliance of the series elastic component $\left(\mathrm{EMD}_{\mathrm{M}-\mathrm{F}}\right)$ in the $\mathrm{VL}, \mathrm{VM}$, and RF contributed equally to $E M D_{\mathrm{E}-}$ F during dynamic muscle actions (isokinetic and dynamic constant external resistance). In addition, after 5 min of recovery the relative contributions from $E M D_{E-M}$ and $E M D_{M-F}$ to $E M D_{E-F}$ for the $V L, V M$, and $R F$ remained similar to those during pretest and posttest measurements.

\section{Conclusion}

In summary, there were fatigue-induced increases in $\mathrm{EMD}_{\mathrm{E}-\mathrm{M}^{\prime}} \mathrm{EMD}_{\mathrm{M}-\mathrm{F}^{\prime}}$ and $E_{\mathrm{E}-\mathrm{F}}$ from the $\mathrm{VL}, \mathrm{VM}$, and RF, however, all EMD measures for all muscles returned to pretest values after 5 min of recovery. Thus, excitation-contraction coupling failure (increased $\mathrm{EMD}_{\mathrm{E}-\mathrm{M}}$ ) and increased compliance of the series elastic component (increased $\mathrm{EMD}_{\mathrm{M}-\mathrm{F}}$ ) were present immediately after the fatiguing protocol, but subsided after 5 min of recovery. In addition, during the pretest, posttest, and 5 min recovery maximal isokinetic knee extension muscle actions there were muscle-specific (VL and VM versus RF) differences in the $\mathrm{EMD}_{\mathrm{E}-\mathrm{M}^{\prime}} \mathrm{EMD}_{\mathrm{M}-\mathrm{F}^{\prime}}$ and $\mathrm{EMD}_{\mathrm{E}-\mathrm{F}}$ measurements. That is, $\mathrm{EMD}_{\mathrm{E}-\mathrm{M}^{\prime}}$ $E M D_{M-F}$ and $E M D_{E-F}$ were greater for the RF than the $V L$ and $V M$. These muscle-specific differences may be associated with differences in muscle architecture (Lieber and Friden 2000). In the current study, there were also similar 
relative contributions from $E M D_{E-M}$ and $E M D_{M-F}$ to $E M D_{E-F}$ from the $V L, V M$, and RF during the pretest, posttest, and 5 min recovery measurements despite changes in the absolute EMD measures. Therefore, fatigue resulted in increases in $E M D_{E-M^{\prime}} E M D_{M-F^{\prime}}$ and $E M D_{E-F}$ from the $V L, V M$, and $R F$, which returned to pretest values after 5 min of recovery.

Acknowledgments - We would like to thank our participants for their time and dedication.

\section{References}

Abe T, Kumagai K and Brechue W 2000 Fascicle length of leg muscles is greater in sprinters than distance runners Med. Sci. Sports Exerc. 32 1125-9

Basmajian J and De Luca C 1985 Muscles Alive: Their Functions Revealed by Electromyography (Baltimore, MD: Williams \& Wilkins) vol 278, p 126

Begovic H, Zhou G, Li T, Wang Y and Zheng Y 2014 Detection of the electromechanical delay and its components during voluntary isometric contraction of the quadriceps femoris muscle Frontiers Physiol. 5 14-9

Ce E, Rampichini S, Agnello L, Limonta E, Veicsteinas A and Esposito F 2013 Effects of temperature and fatigue on the electromechanical delay components Muscle Nerve 47 566-76

Ce E, Rampichini S and Esposito F 2015a Novel insights into skeletal muscle function by mechanomyography: from the laboratory to the field Sport Sci. Health 11 1-28

Ce E, Rampichini S, Limonta E and Esposito F 2014 Fatigue effects on the electromechanical delay components during the relaxation phase after isometric contraction Acta Physiol. 211 82-96

Ce E, Rampichini S, Venturelli M, Limonta E, Veicsteinas A and Esposito F 2015b Electromechanical delay components during relaxation after voluntary contraction: reliability and effects of fatigue Muscle Nerve 51 907-15

Chan A Y, Lee F, Wong P, Wong C and Yeung S 2001 Effects of knee joint angles and fatigue on the neuromuscular control of vastus medialis oblique and vastus lateralis muscle in humans Eur. J. Appl. Physiol. 84 36-41

Conchola E, Thompson B and Smith D 2013 Effects of neuromuscular fatigue on the electromechanical delay of the leg extensors and flexors in young and old men Eur. J. Appl. Physiol. 113 2391-9

Conchola E C, Thiele R, Palmer T, Smith D and Thompson B 2015 Effects of neuromuscular fatigue on electromechanical delay of the leg extensors and flexors in young men and women Muscle Nerve 52 844-51

Costa P, Ryan E, Herda T, Walter A, Hoge K and Cramer J 2012 Acute effects of passive stretching on the electromechanical delay and evoked twitch properties: a gender comparison J. Appl. Biomech. 28 645-54

Cybexll 1991 Cybex II User Manual: Testing and Rehabilitation Guide (New York: Division of Lumex, Ronkonkoma) 
de Ste Croix M, Elnagar Y, Iga J, James D and Ayala F 2015 Electromechanical delay of the hamstrings during eccentric muscle actions in males and females: implications for non-contact ACL injuries J. Electromyogr. Kinesiol. 25 901-6

Esposito F 2013 Reliability of the electromechanical delay components assessment during the relaxation phase Physiol. J. 2013517838

Hakkinen K and Komi P 1983 Electromyographic and mechanical characteristics of human skeletal muscle during fatigue under voluntary and reflex conditions Electroencephalogr. Clin. Neurophysiol. 55 436-44

Hannah R, Minshull C, Smith S and Folland J 2014 Longer electromechanical delay impairs hamstrings explosive force versus quadriceps Med. Sci. Sports Exerc. 46 963-72

Hermens H, Freriks B, Merletti R, Stegeman D, Blok J, Rau G, Disselhorst-Klug C and Hagg G 1999 European recommendations for surface electromyography Roessingh Res. Dev. 8 13-54

Hopkins J, Feland J and Hunter I 2007 A comparison of voluntary and involuntary measures of electromechanical delay Int. J. Neurosci. 117 597-604

Howatson G, Glaister M, Brouner J and Van Someren K 2009 The reliability of electromechanical delay and torque during isometric and concentric isokinetic contractions J. Electromyogr. Kinesiol. 19 975-9

Lacourpaille L, Nordez A and Hug F 2013 Influence of stimulus intensity on electromechanical delay and its mechanisms J. Electromyogr. Kinesiol. 23 51-5

Lieber R and Friden J 2000 Functional and clinical significance of skeletal muscle architecture Muscle Nerve 23 1647-66

Minshull C, Eston R, Bailey A, Rees D and Gleeson N 2012 Repeated exercise stress impairs volitional but not magnetically evoked electromechanical delay of the knee flexors J. Sport Sci. 30 217-25

Norman R and Komi P 1979 Electromechanical delay in skeletal muscle under normal movement conditions Acta Physiol. Scand. 106 241-8

Orizio C, Esposito F, Paganotti I, Marino L, Rossi B and Veicsteinas A 1997 Electrically-elicited surface mechanomyogram in myotonic dystrophy Ital. J. Neurol. Sci. 18 185-90

Rampichini S, Ce E, Limonta E and Esposito F 2014 Effects of fatigue on the electromechanical delay components in gastrocnemius medialis muscle Eur. J. Appl. Physiol. 114 639-51

Smith C et al 2016a Electromyographic responses from the vastus medialis during isometric muscle actions Int. J. Sports Med. 37 647-52

Smith C, Housh T, Hill E, Johnson G and Schmidt R 2016b Changes in electromechanical delay during fatiguing dynamic muscle actions Muscle Nerve 56 315-20

Smith C, Housh T, Hill E, Johnson G and Schmidt R 2017 Dynamic versus isometric electromechanical delay in non-fatigued and fatigued muscle: a combined electromyographic, mechanomyographic, and force approach J. Electromyogr. Kinesiol. 33 34-8

Stock M, Olinghouse K, Mota J, Drusch A and Thompson B 2015 Muscle group specific changes in the electromechanical delay following short-term resistance training J. Sci. Med. Sport 19 761-5 
Taylor A, Bronks R, Smith P and Humphries B 1997 Myoelectric evidence of peripheral muscle fatigue during exercise in severe hypoxia: some references to $\mathrm{m}$. vastus lateralis myosin heavy chain composition Eur. J. Appl. Physiol. 75 151-9

Vos E J, Harlaar J and Van Ingen S 1991 Electromechanical delay during knee extensor contractions Med. Sci. Sport Exerc. 23 1187-93

WMA 2013 World Medical Association Declaration of Helsinki: ethical principles for medical research involving human subjects JAMA 310 2191-4

Zhou S, Carey M, Snow R, Lawson D and Morrison W 1998 Effects of muscle fatigue and temperature on electromechanical delay Electroencephalogr. Clin. Neurophysiol. 3867 